\title{
État social et mondialisation : analyse juridique des solidarités
}

\section{Alain Supiot}

\section{(2) OpenEdition}

1 Journals

Édition électronique

URL : https://journals.openedition.org/annuaire-cdf/15336

DOI : $10.4000 /$ annuaire-cdf.15336

ISBN : 978-2-7226-0572-5

ISSN : 2109-9227

Éditeur

Collège de France

Édition imprimée

Date de publication : 30 décembre 2020

Pagination : 475-490

ISBN : 978-2-7226-0516-9

ISSN : 0069-5580

\section{Référence électronique}

Alain Supiot, «État social et mondialisation : analyse juridique des solidarités », L'annuaire du Collège de France [En ligne], 118 | 2020, mis en ligne le 01 avril 2021, consulté le 22 août 2022. URL : http:// journals.openedition.org/annuaire-cdf/15336 ; DOI : https://doi.org/10.4000/annuaire-cdf.15336 


\title{
ÉTAT SOCIAL ET MONDIALISATION : ANALYSE JURIDIQUE DES SOLIDARITÉS
}

\author{
Alain SUPIOT \\ Professeur au Collège de France
}

Mots-clés : entreprise, institution, démocratie économique, modèles sociaux, droit social, personnel, négociation collective

Le colloque "Revisiter les solidarités en Europe » est disponible en vidéo sur le site internet du Collège de France (https://www.college-de-france.fr/site/alain-supiot/ symposium-2017-2018.htm).

\section{ENSEIGNEMENT}

COURS - FIGURES JURIDIQUES DE LA DÉMOCRATIE ÉCONOMIQUE (II)

\section{Résumé}

Le cours de l'année 2017-2018 a été consacré à la question de la démocratisation de l'entreprise. Sa première partie a cherché à saisir juridiquement la notion d'entreprise, en analysant les difficultés de sa définition, en retraçant sa généalogie institutionnelle et en revisitant les théories dont elle a fait l'objet en droit social. La seconde partie du cours a traité des conditions d'une démocratisation de l'entreprise, en analysant la tension normative dont elle est aujourd'hui l'objet et la dynamique de son autoréglementation.

\section{Introduction}

La soumission croissante des États à l'influence des détenteurs du pouvoir économique les conduit à transférer aux entreprises le soin de gérer ou réglementer des questions d'intérêt général. Du point de vue juridique, ce renoncement se traduit par l'affermage de fonctions d'intérêt général aux entreprises privées, ainsi que par la promotion par les pouvoirs publics de la responsabilité sociale et environnementale 
des entreprises (RSE), où la Commission et le Conseil européen voient la contrepartie «d'un environnement réglementaire plus favorable aux entreprises ». La promotion d'un tel échange entre déréglementation publique et autoréglementation des entreprises soulève deux questions. La première est de savoir si et jusqu'à quel point les entreprises transnationales peuvent exercer un pouvoir normatif susceptible de compléter ou de concurrencer celui des États. La seconde est de savoir s'il est possible de faire de l'entreprise l'un des cadres de la démocratie économique.

\section{La définition juridique de l'entreprise}

La liberté d'entreprendre est en France un principe constitutionnel fondé, non sur l'article 17 de la Déclaration de 1789 relative au droit de propriété, mais sur son article 4 définissant la liberté comme le «pouvoir de faire tout ce qui ne nuit pas à autrui ». L'entreprise est ainsi située du côté du faire, de l'agir, et non pas de l'avoir ou de l'être. Les entreprises peuvent toutefois, à l'instar des Etats, se faire reconnaître comme des personnes morales, c'est-à-dire comme des sujets de droit d'un type particulier, dont on postule qu'à l'égal des hommes ils sont animés d'une volonté propre, mais qui échappent en revanche à leur mortelle condition.

D'où la polysémie de la notion d'entreprise, qui désigne non seulement l'action économique, mais aussi les personnes morales créées pour les besoins de cette action. Cette polysémie est la source de constantes confusions et un obstacle majeur à la mise en œuvre de la responsabilité des dirigeants des entreprises. Lorsque la liberté d'entreprendre s'exerce sous le masque de personnes morales dont la responsabilité est limitée, comment et à qui identifier l'entreprise : à ces personnes morales; aux groupes ou réseaux qu'elles constituent; ou bien aux personnes physiques qui les contrôlent financièrement?

La question se pose bien sûr en matière d'imputation de responsabilité et d'identification de l'employeur. Mais elle se pose aussi de façon beaucoup plus élémentaire, lorsqu'il s'agit de dénombrer les entreprises pour construire l'image statistique de l'économie. Aussi le règlement $n^{\circ}$ 696/93 du Conseil européen a-t-il imposé une définition commune de l'entreprise comme étant «la plus petite combinaison d'unités légales qui constitue une unité organisationnelle de production de biens et de services jouissant d'une certaine autonomie de décision ». Ainsi définie comme une entité économique caractérisée par un pouvoir décisionnaire s'exerçant le cas échéant sur une combinaison d'unités légales, la notion d'entreprise est un lit de Procuste, sur lequel on trouve pêle-mêle des entreprises publiques et privées, avec ou sans but lucratif, avec ou sans salariés, avec ou sans personnalité morale. Il en va de même des définitions adoptées par la jurisprudence européenne en droit de la concurrence, selon laquelle l'entreprise est une «entité exerçant une activité économique », l'activité économique est celle que les entreprises exercent sur un marché, et le marché est le point de rencontre de la demande et de l'offre d'acteurs économiques... Sous le vernis du vocabulaire économique, cette définition tautologique et autoréférentielle de l'entreprise relève de la dogmatique juridique, et permet à la Cour de justice d'étendre à son gré l'empire des libertés économiques garanties par les traités européens. Cette fluidité se retrouve dans la directive « transfert » 2001/23/CE qui subsume, sous la même définition, les entreprises, les établissements, les parties d'entreprises ou d'établissements, autrement dit qualifie d'entreprise aussi bien un tout que chacune de ses parties. 
Dès lors, céder une entreprise c'est céder non seulement un droit réel sur des choses corporelles ou incorporelles, mais aussi un pouvoir de direction sur les hommes et les femmes qui se trouvent ainsi juridiquement attachées à ces choses et constituent son «personnel». Cet ensemble organisé de choses et de personnes a une identité qui se conserve par-delà le renouvellement constant de ces choses et de ces personnes. De ce point de vue, le personnel occupe une place comparable à celle du peuple, du demos, dans la sphère politique, qui lui aussi se renouvelle constamment au sein d'un organisme politique conservant son identité. Cette permanence du personnel au-delà du changement des personnes et sa soumission à un même pouvoir sont autant de traits qui permettent d'affirmer que l'entreprise, dès lors que son activité dépend du travail de plusieurs personnes, n'est pas seulement une entité économique, mais aussi une entité politique. Mais peut-on pour autant y transposer les catégories de pensée de la démocratie ? Pour répondre par l'affirmative, il faut commencer par admettre que l'entreprise est plus que l'exercice d'une liberté économique : une institution.

\section{Généalogie institutionnelle de l'entreprise}

Aux origines institutionnelles de l'entreprise se trouve l'oikos grec ou la domus romaine, c'est-à-dire la maisonnée comme communauté économique et lignagière, soumise au pouvoir du pater familias. Le patronat est ainsi une notion héritée du droit romain, où le patronus désignait l'ancien maître d'un esclave affranchi, auquel ce dernier demeurait lié par une obligation de respect (obsequium) et de services (operce), ainsi que par des liens d'obligation alimentaire et successoraux (bona). La sphère de l'oikos, dans son sens originel comme dans le sens moderne de l'oikosnomos, de l'éco-nomie, constitue ce que Georges Gurvitch appelle un «fait normatif » : son existence est antérieure et à certains égards indépendante de la loi de la cité et de l'hétéronomie de l'État, car elle engendre ses propres normes, elle est auto-nome.

Jusqu'à la fin du Moyen Âge, les associations humaines de ce type ont été pensées sur le modèle de l'ecclesia chrétienne, c'est-à-dire comme autant de corps mystiques qui, en dernière instance, tirent leur identité et leur cohérence d'une foi, d'une fides partagée par leurs membres; de même que la notion d'ecclesia, cette référence à la fides, était un héritage de l'Antiquité classique (selon Cicéron, le fondement de la justice est la fides, c'est-à-dire le respect de notre parole et l'inviolable fidélité à nos engagements).

Grotius (1583-1645), l'un des pères du droit international, fit le premier l'économie de cette référence religieuse. Dans une Europe déchirée par les guerres de religion, le droit et les institutions ne pouvaient plus être adossés à la commune référence religieuse, à la figure du pontife, qui avait permis l'essor de la lex mercatoria médiévale. Pour que le commerce national, mais surtout international, puisse se déployer, il fallait donner au droit un fondement susceptible d'être reconnu par tous, indépendamment des appartenances religieuses : l'appetitus societatis, le désir de société ou sociabilité, qui serait inhérent à la nature humaine.

Les règles que Grotius prétend ainsi déduire de la nature humaine dérivent en fait directement de sa culture juridique, qui est celle d'un juriste de la bourgeoisie protestante, rompu au droit romain et au droit canon. Avocat de la Compagnie néerlandaise des Indes orientales, il prétend ainsi fonder sur le droit naturel la liberté du commerce, quand bien même elle contrevenait à la souveraineté des États. Grotius 
posait ainsi dès 1609 le fondement dogmatique de la globalisation marchande, que la science économique a repris à son compte

Sa découverte de la dimension normative inhérente à la sociabilité humaine conduit aussi Grotius à reconnaître l'existence, à l'intérieur même d'un État, d'associations humaines capables de s'autoréglementer, au premier rang desquelles la famille et l'entreprise. Ces associations infra-étatiques sont selon lui nécessairement hiérarchiques : leur droit procède de la volonté d'un seul (le père ou le patron), dans les limites toutefois du respect de l'ordre juridique étatique. L'idée que les associations économiques puissent, comme la société internationale, être des associations entre égaux ne lui traverse pas l'esprit, de même qu'il n'envisage pas qu'elles puissent être émancipées de la tutelle d'un État.

C'est chez Leibniz (1646-1716) que l'on trouve nettement affirmée pour la première fois la théorie d'associations infra-étatiques capables de s'autoréglementer sur un mode coopératif. Sa philosophie du droit va de pair avec son œuvre mathématique, notamment l'invention du calcul infinitésimal, et s'accorde aussi avec sa monadologie qui, contrairement au mécanisme cartésien, représente le monde comme une unité résultant de l'agrégation de monades toutes différentes et mues chacune par ses forces propres. De même, les associations humaines ne sont ni des agrégats d'individus, ni des entités transcendantes, mais des milieux vitaux, qui tout à la fois nourrissent et se nourrissent de la diversité de leurs membres. La dichotomie aujourd'hui devenue lieu commun, qui oppose l'individu et la société, n'a de ce point de vue aucun sens.

Contrairement à Hobbes, Leibniz ne pense pas que le droit procède de la volonté d'individus souverains, qui ne tiennent ensemble qu'en obéissant à la volonté prépondérante d'un super individu : l'État. Fidèle à la tradition germanique, il voit dans la société une association de communautés, de Gemeinschaften, ce qui le conduit à distinguer le ius societatis et le ius proprietatis. Ce dernier est fondamentalement un ius belli, un droit de la guerre (économique) et n'a vocation à régir que les rapports des hommes avec les choses, car il est impuissant à régir la société en tant que communauté humaine. C'est donc un contresens d'appliquer ce ius proprietatis aux liens sociaux, qui relèvent du ius societatis seu congruentium (droit de la société ou de la concordance), c'est-à-dire du droit social qui a pour objet la collaboration des hommes et oblige chacun à concourir au bien de tous.

On touche ici du doigt les longues racines historiques de la divergence toujours actuelle entre les manières anglaise et allemande de concevoir l'entreprise. Côté anglais, l'entreprise est saisie comme un bien, soumis au ius proprietatis des actionnaires et dont les salariés sont des fournisseurs parmi d'autres, qui usent de la propriété supposée de leur propre corps. Côté allemand, l'entreprise est en revanche saisie comme une manifestation du ius societatis, une communauté poursuivant ses intérêts propres, auxquels se trouvent subordonnés les intérêts particuliers des actionnaires comme ceux des salariés.

Parmi les héritiers de Leibniz, il faut retenir Otto von Gierke (1841-1921) dont l'œuvre monumentale est incontournable pour qui veut comprendre la conception «rhénane» de l'entreprise. Selon Gierke, le droit social (Sozialrecht: il est le premier à forger ce concept dans son sens large) peut être de type égalitaire et coopératif (Genossenschaftsrecht) ou au contraire autoritaire et hiérarchique (Herrschaftsrecht). Le Genossenchaftsrecht ainsi théorisé par Gierke a été le cadre théorique dans lequel ont pu ensuite éclore la notion de démocratie économique (Wirtschaftsdemokratie) sous la république de Weimar, puis, après 1945, la cogestion 
des entreprises (Mitbestimmung) et l'économie sociale de marché. Le véritable titulaire de la propriété étant la personne morale, son exercice doit être placé sous le contrôle conjoint de représentants de tous ses membres, c'est-à-dire dans le cas d'une entreprise aussi bien des salariés que des employeurs.

- Cette généalogie institutionnelle de l'entreprise s'est achevée par l'évocation de Proudhon, le père de l'idée de démocratie industrielle. Pour l'établir, il faut instaurer, en regard de la constitution politique, une constitution économique, de type fédératif et mutuelliste, qui garantisse à chaque type d'association la possibilité de se gouverner elle-même. Ainsi entendue, la mutualité n'exclut ni la propriété privée ni l'État, mais elle permet de contrebalancer les risques symétriques de l'imperium de l'État et du dominium du propriétaire, qui livrés à eux-mêmes condamnent le plus grand nombre à l'état de subordination. Prolongées par les tenants du solidarisme, ces vues ont profondément marquées le modèle social français, comme le montrent par exemple l'adoption de la charte d'Amiens dans le domaine syndical, mais aussi le droit de la mutualité ou la place de la démocratie sociale dans le plan de sécurité sociale adopté en 1945.

\section{Théories juridiques de l'entreprise}

Depuis la fin du XIX $\mathrm{X}^{\mathrm{e}}$ siècle, la conception de l'entreprise en droit français a été marquée par l'œuvre de quelques grands juristes, dont la connaissance est indispensable à la compréhension des débats contemporains. Le premier est Léon Duguit (1859-1928), dont la conception fédéraliste de l'État comme «coopération de services publics » se situe clairement dans l'héritage proudhonien.

Raymond Saleilles (1855-1912) a opéré en droit privé une révolution doctrinale comparable à celle de Duguit en droit public et l'invention de l'État-providence n'aurait pas été fonctionnelle sans ses théories du contrat d'adhésion et de la responsabilité pour risques, qui tiraient en droit privé toutes les conséquences de la domination économique et juridique exercée sur les salariés : «Le XIX siècle finissant a entrevu - écrit-il - un idéal de démocratie sociale, auquel la démocratie politique aura servi d'étape ${ }^{1} »$. Sa théorie de la personnalité juridique évite de sombrer dans l'anthropomorphisme qui conduit de nos jours la jurisprudence à étendre les droits de l'homme aux personnes morales en général et aux sociétés commerciales en particulier. À la différence de la personnalité juridique reconnue à tout être humain, qui procède de ce qu'il est en lui-même une fin en soi, celle d'un groupement humain (association ou fondation) ne vaut qu'au regard et dans les limites de la légitimité sociale des buts poursuivis par ce groupement. La «fondamentalisation» des droits de l'entreprise observée depuis le tournant néolibéral méconnaît cette nécessaire interprétation fonctionnelle de la personnalité juridique des sociétés commerciales, qui ne devraient se voir reconnaître de droits fondamentaux que dans la mesure où elles poursuivent la réalisation d'une œuvre ou d'une entreprise socialement utile, distincte du simple enrichissement de leurs actionnaires.

Cette idée d'œuvre ou d'entreprise est la première des trois composantes de la célèbre définition que Maurice Hauriou (1856-1929) donne de l'institution. La deuxième est un pouvoir organisé et la troisième ce qu'Hauriou désigne comme des

1. R. SALEILLES, «Le Code civil et la méthode historique», in Le Code civil (1804-1904). Livre du centenaire, t. 1, 1904, p. 116. 
«manifestations de communion réglées par des procédures ». Ces manifestations peuvent consister par exemple en acclamations, réunions, chants ou remises de distinctions, qui donnent aux membres de l'institution le «sentiment de leur émotion commune " (où l'on retrouve donc l'idée de fides, tantôt évoquée). Dans une institution, au sens ainsi défini par Hauriou, c'est la réalisation de l'œuvre qui tout à la fois justifie et borne le pouvoir des dirigeants.

Tous les auteurs qui ont ainsi mis en évidence, au-delà du positivisme juridique, l'existence d'institutions générant leur propre droit, ont été amenés à récuser les dichotomies individu/société, État/société ou État/marché, qui empêchent de penser comme un même phénomène l'institution des hommes par leurs associations et l'institution de ces associations par les hommes. C'est le cas également aux ÉtatsUnis de John Dewey (1859-1952), selon qui ces dichotomies ont pour effet de substituer des abstractions irréelles à l'expérience sociale et de masquer ainsi la réduction du champ de la démocratie par l'essor de grandes entreprises, dont le principe de fonctionnement est la domination.

Ce tour d'horizon des théories juridiques de l'entreprise s'est achevé par l'étude de celle de Paul Durand (1908-1960), qui a marqué toute la doctrine juridique française de l'après-guerre. Reposant tout entière sur une analogie de l'entreprise et de la société politique, cette théorie devait nécessairement faire une certaine place à la démocratie. Mais comme elle devait aussi rendre compte de l'état du droit positif, il s'agit d'une place résiduelle, ou plus exactement d'un germe de démocratie dont Durand pensait qu'il pourrait éclore avec les progrès ultérieurs du droit du travail.

Aujourd'hui, l'entreprise-institution visée par Hauriou ou Durand se lézarde et laisse place à ce que Charley Hannoun a justement appelé l'«entreprisemarchandise », dont on postule (juridiquement à tort) qu'elle est la propriété exclusive des actionnaires. Le démembrement des entreprises en unités légales distinctes permet de concentrer les profits et d'externaliser les coûts. Le point de fuite de cette évolution est la holding financière, entreprise qui ne produit rien (sinon de l'argent), n'emploie quasiment personne, et contrôle en fait un réseau d'entreprises vassales chargées des tâches de production, sans avoir en droit à en assumer les responsabilités. Cette tendance lourde prend à revers le processus d'institutionnalisation de l'entreprise.

\section{Les antinomies du droit de l'entreprise}

Le droit de l'entreprise est aujourd'hui le théâtre de deux tendances contradictoires. D'un côté, l'entreprise est réduite à une marchandise, à une chose dans le commerce, livrée à la spéculation des marchés financiers et dont le propriétaire peut disposer de façon discrétionnaire. De l'autre, l'entreprise est traitée comme une institution écologiquement et socialement responsable, capable non seulement de secréter son propre droit de façon plus ou moins démocratique, mais aussi de prendre en charge les missions d'intérêt général que les États ne parviennent plus à remplir dans le contexte de darwinisme normatif imposé par la globalisation. Cette contradiction sera à terme intenable. Mais elle est masquée par la promotion de l'autoréglementation de l'entreprise, qui a la vertu de répondre en même temps à la demande de déréglementation du marché du travail et à l'aspiration à plus de démocratie et de responsabilité dans l'entreprise.

La démocratisation de l'entreprise ne doit pas être confondue avec la démocratie sociale. Cette dernière a pour base juridique la liberté syndicale - consacrée 
notamment par l'alinéa 6 du préambule de la Constitution de 1946 - et pour premier objet l'organisation collective des salariés et la négociation collective avec les employeurs ou leurs organisations. La démocratie sociale ainsi entendue s'exerce aux niveaux professionnel et interprofessionnel, plus rarement au niveau territorial. À la différence de la démocratie par le nombre, qui domine la représentation politique, la démocratie sociale est essentiellement qualitative et consiste à permettre la représentation de différents groupes d'intérêts ayant de la société et de ses problèmes une expérience concrète et des points de vue différents. Elle vise notamment à compenser l'absence quasi-totale de parlementaires issus des classes populaires. Ainsi qu'en témoigne par exemple la loi Larcher (2007), la démocratie sociale ne signifie pas le retour du pouvoir réglementaire des corporations, mais l'englobement de la représentation des intérêts collectifs dans la définition de l'intérêt général, le Parlement devant s'inspirer des résultats des négociations interprofessionnelles mais conservant toujours le droit du dernier mot.

La démocratisation de l'entreprise procède quant à elle du «droit à la participation », consacré par l'alinéa 8 du préambule de la Constitution de 1946 . Ce droit vise une démocratisation du pouvoir économique dans l'entreprise qui ne se confond pas mais s'ajoute à la démocratie sociale. Ce n'est qu'à partir de l'institution en 1968 de délégués syndicaux dans les entreprises que ces dernières sont devenues à leur tour un niveau de négociation, mais un niveau secondaire qui, jusqu'aux réformes Auroux de 1982, est demeuré lié par le principe de faveur et ne pouvait donc servir qu'à améliorer les droits que les salariés tiraient de la loi ou de la convention de branche.

La citoyenneté des salariés dans l'entreprise mise en avant par ces réformes s'est heurtée, tout comme le droit de participer à sa gestion, à la jurisprudence Brinon (1954), qui estime «l'employeur seul juge des circonstances qui le déterminent à cesser son exploitation ». En écartant dans sa décision «Loi Florange » toute idée de contrôle du détournement de pouvoir de l'employeur qui décide de fermer une entreprise plutôt que d'accepter une offre de reprise sérieuse, le Conseil constitutionnel a confirmé ce principe de «l'employeur seul juge ».

Le pouvoir discrétionnaire ainsi conféré à ses dirigeants interdit de voir dans l'entreprise privée une institution, animée par une œuvre à accomplir. Il en va différemment dans les entreprises ou services publics, dont les dirigeants exercent un pouvoir fonctionnel, justifié et limité par les nécessités de cet accomplissement. Cette distinction tend toutefois à s'estomper avec l'assujettissement des entreprises publiques à de purs objectifs de rentabilité financière. Les dérives inhérentes au new public management, montrent qu'un service public peut, tout comme une entreprise privée, succomber à la marchandisation et se condamner ainsi à une lente désinstitution. Le vice de conception du new public management n'est donc pas de considérer les services publics comme des entreprises, mais d'ignorer l'idée d'œuvre qui donne sa dimension institutionnelle à l'entreprise, et de réduire les buts de celle-ci à la maximisation de résultats chiffrés.

Le droit européen a été à l'évidence l'un des moteurs de cette assimilation de l'intérêt collectif à la mise en concurrence d'agents poursuivant leurs intérêts individuels. Mais elle est aussi en France le fait du Conseil d'État, converti à l'idée que la clé de voûte de la réalisation de l'intérêt général est la concurrence, comme le montre par exemple l'évolution de sa jurisprudence relative à l'extension d'une convention collective de branche. L'incorporation par l'État des valeurs du secteur privé ne retient donc de ce dernier que l'impératif concurrentiel et non pas l'idée de 
solidarité autour d'une œuvre commune, qui est pourtant la clé de voûte de toute institution et l'une des caractéristiques des entreprises les plus durables. Sont ainsi étendus au secteur public les facteurs de désinstitution de l'entreprise qui, dans le privé, accompagnent sa financiarisation.

Corollaire de cette financiarisation, la désinstitution de l'entreprise privée rend illusoire sa démocratisation. Car la démocratie, en tant qu'assemblée de paroles, se caractérise par la référence commune à un intérêt commun qui transcende les intérêts particuliers. Le projet de modification de la définition des sociétés commerciales qui se référerait à la « raison d'être » de l'entreprise et non seulement à la réalisation de bénéfices - témoigne toutefois d'une prise de conscience des risques inhérents à cette désinstitution.

\section{La dynamique de l'autoréglementation de l'entreprise au prisme de la diversité des modèles sociaux}

En donnant une portée générique aux accords dérogatoires, la loi Auroux du 13 novembre 1982 avait enfoncé un premier coin dans le pilier central du droit du travail français : la notion d'ordre public social. S'engouffrant dans cette brèche, les milieux patronaux n'ont cessé de réclamer, depuis les années 1980, la création d'un « contrat collectif d'entreprise » ayant vocation à devenir la source première du droit du travail. Depuis la loi Fillon de 2004 jusqu'aux ordonnances Macron de 2017, le législateur s'est efforcé de mettre ce projet en œuvre, en étendant régulièrement le champ d'application des accords dérogatoires. La pratique pourtant n'a pas suivi et les entreprises s'en sont massivement tenues aux dispositions conventionnelles de branche.

Mais cette extension, puis la primauté de principe donnée à ces accords sur les conventions de branche, n'en ont pas moins obligé à reconsidérer les conditions de la négociation d'entreprise, sous la double contrainte, d'une part d'assurer la légitimité de ces conventions et d'autre part d'en permettre la conclusion dans les entreprises dépourvues de représentation syndicale. Contraintes largement contradictoires, puisque la première conduit à renforcer les conditions de capacité à conclure ces accords, tandis que la seconde conduit au contraire à les assouplir. D'un côté, on a subordonné la capacité de conclure les accords d'entreprise à la condition que les syndicats signataires aient réuni une majorité de voix lors des élections professionnelles ; et de l'autre, on a conféré cette capacité à des salariés n'ayant pas de mandat de délégué syndical.

Répondant aux appels répétés de l'Union européenne et de l'OCDE à des «réformes structurelles» du marché du travail, les ordonnances Macron de septembre 2017 ont accentué ce mouvement, en tentant de faire de l'entreprise un ordre juridique autonome, i.e. capable de se doter de ses propres règles indépendamment des dispositions conventionnelles de branche. Posé par le nouvel article L. 2253-3 du Code du travail, ce renversement de la hiérarchie des normes conventionnelles fait mécaniquement refluer la démocratie sociale, telle qu'elle s'exerce au sein des branches professionnelles.

Ce reflux de la démocratie sociale n'a pas pour contrepartie un minimum de démocratisation dans l'entreprise, mais bien plutôt un progrès des liens d'allégeance. En effet, au plein sens du terme - celui d'une assemblée de citoyens placés sur un pied d'égalité pour délibérer de leur bien commun - la démocratie dans l'entreprise n'est guère envisageable que dans le cadre des coopératives, dont les travailleurs sont 
aussi les associés. À cette exception près, le personnel est par définition dans un état de subordination juridique et de dépendance économique vis-à-vis de l'employeur. Sa représentation doit être ancrée dans la représentation syndicale hors de l'entreprise, car seul cet ancrage peut garantir à l'intérieur de l'entreprise un certain équilibre des forces, sans lequel elle demeure un ordre autocratique. Pour que la primauté de principe dorénavant attribuée aux accords d'entreprise puisse être un facteur de démocratisation de celle-ci, il faudrait créer les conditions de cet équilibre. Il n'existe pas à cette fin un seul modèle, mais plusieurs, ainsi que le montre le droit comparé.

Si la voie empruntée était celle du modèle allemand, cela conduirait à une profonde réforme de notre droit de l'entreprise, dans ses deux volets de droit des sociétés et de droit du travail. Le modèle social «rhénan » est historiquement l'une des variantes de la culture juridique allemande, qui conçoit la société comme une association de communautés organisées (Gemeinschaften), coordonnées par l'État. Au cours de l'histoire, cette culture communautaire a oscillé entre, d'une part, une version autoritaire, illustrée par Bismarck puis - sous une forme paroxysmique - par le Führerprinzip, appliqué par le nazisme aux entreprises ; et, d'autre part, une version démocratique, déjà théorisée, nous l'avons vu, chez Leibniz, puis au XIX ${ }^{\mathrm{e}}$ siècle dans la grande ouvre de Gierke, mais systématisé en droit du travail par Sinzheimer au début du $\mathrm{XX}^{\mathrm{e}}$ siècle. La notion de démocratie économique élaborée par Sinzheimer a inspiré les dispositions de la Constitution de Weimar, prévoyant une collaboration sur un pied d'égalité des salariés et des employeurs au sein de différentes communautés professionnelles organisées. C'est à cette conception démocratique que l'Allemagne est revenue après l'effondrement du nazisme, en instaurant en 1952 la codétermination (Mitbestimmung) dans les grandes entreprises au lieu de les nationaliser comme on le fit en France.

La codétermination - i.e. le gouvernement de l'entreprise par un directoire œuvrant sous le double contrôle des représentants des actionnaires et des salariés n'est toutefois que l'un des trois pieds sur lesquels repose le modèle social allemand. Le deuxième est la représentation du personnel dans des comités d'établissement chargés d'une mission de coopération mais aussi en certains domaines d'un pouvoir de codécision. Et le troisième la négociation collective de branche, qui est et demeure l'attribut exclusif des organisations syndicales. Le droit allemand veille ainsi à garantir dans les relations collectives de travail un équilibre des forces, sans lequel la négociation collective ne peut que dégénérer en soumission collective. Ce rééquilibrage met en jeu au moins cinq balances :

- entre capital et travail ;

- entre représentation élective et représentation syndicale ;

- entre collectivité de travail et direction;

- entre entreprise et branche professionnelle ou territoriale ;

- entre loi et négociation collective.

Ce n'est pas la détérioration du sort des chômeurs et des salariés précaires, induite par les réformes Hartz au début des années 2000, qui explique le «miracle économique allemand », mais cette culture juridique communautaire, aujourd'hui combinée à l'avantage comparatif d'un euro sous-évalué et à la sous-traitance dans les pays postcommunistes.

L'invocation de l'exemple allemand pour justifier les réformes du Code du travail français est donc fallacieuse. À aucun moment il n'a été question de réformer le système de représentation et de négociation collective en prenant en compte la 
structure d'ensemble de ce modèle et les équilibres qu'il suppose d'instituer. Rien dans ces réformes ne s'approche, même de loin, de l'idée de codétermination. Et l'un de leurs axes a été d'affaiblir autant que possible les conventions collectives de branche, qui demeurent le pivot du droit du travail allemand.

Les réformes françaises pourraient davantage être rapprochées de l'expérience du Royaume-Uni. Le modèle britannique d'industrial relations reposait non sur l'interventionnisme législatif, mais sur la recherche d'un équilibre des forces agissant sur le marché du travail. Historiquement, l'objectif des syndicats britanniques a été d'empêcher que les juges ne viennent disqualifier les collective agreements qu'ils arrachaient aux employeurs, et qui étaient sans valeur au regard de la common law. Ainsi fondé sur des immunités juridiques garanties par la loi, le modèle britannique a évolué au gré des alternances politiques entre des périodes de flux et de reflux de ces immunités. La période de reflux ouverte par l'arrivée au pouvoir de Thatcher en 1979 a conduit à démanteler de larges pans de ce système. Au point que le gouvernement conservateur lui-même s'inquiète depuis le Brexit de la dislocation du statut salarial, des effets ravageurs de la flexibilité à sens unique (unfair one side flexibility) et de la montée alarmante du nombre de travailleurs pauvres. Au nom d'une « better corporate governance ». La Première ministre Theresa May avait aussi manifesté l'intention d'introduire des représentants des salariés dans les organes de direction des entreprises pour limiter l'entre-soi et cantonner la cupidité et le court-termisme de leurs membres. Mais il semble bien que la résistance des intéressés ait eu raison de sa détermination politique et que cette idée soit abandonnée.

Face à ces modèles allemand et anglais, le droit du travail français demeure dominé par la dichotomie privé/public. Son histoire est celle d'une oscillation entre des périodes où prévaut l'interventionnisme de l'État, qui prétend régenter les relations de travail dans leurs moindres détails, et des périodes de retrait de l'État, où prévaut la doctrine inverse, de liberté contractuelle et de laisser-faire. Cette conception politique réfère toujours la relation de travail à une question de pouvoir, plutôt qu'à une question d'échange marchand comme en Grande-Bretagne ou de communautés de travail comme en Allemagne. Les ordonnances Macron s'inscrivent dans cette conception des relations de travail, en traitant l'entreprise comme une petite société politique dont la loi interne doit pouvoir s'affirmer non seulement visà-vis de la loi professionnelle ou de la loi de l'État, mais aussi vis-à-vis de la force obligatoire des contrats individuels. Sur ce point, elles différent profondément des lois Auroux, qui envisageaient elles aussi l'entreprise comme une société politique, mais avec l'intention d'y faire reconnaître la citoyenneté des travailleurs, et donc leurs droits et libertés individuelles et collectives. Cet objectif, qui aurait pu se réclamer aujourd'hui des mutations du salariat et de l'aspiration à une autonomie plus grande dans le travail, est absent des ordonnances qui visent au contraire à accroître le pouvoir normatif de l'employeur.

Techniquement, des accords par lesquels une partie faible renonce à certains de ses droits en contrepartie de la promesse de la partie forte de lui assurer une certaine sécurité économique constituent en effet des actes d'allégeance. Et ce sont bien en fin de compte des mécanismes d'allégeance collective et individuelle qu'ont institués les ordonnances Macron, comme le montrent notamment leurs dispositions relatives aux entreprises dépourvues de délégués syndicaux ou le recul de la force obligatoire du contrat individuel de travail face aux dispositions moins favorables d'un accord d'entreprise. 


\section{Conclusion}

L'invention de l'État social a permis dans un premier temps de sauver la démocratie politique aussi bien de la dictature du prolétariat que de la prise de contrôle par ce que Roosevelt nommait, pour le comparer aux organisations criminelles, "l'argent organisé ». Mais les modèles sociaux qui avaient permis de fonder sur la liberté syndicale des formes nouvelles de démocratie économique ou sociale sont aujourd'hui à leur tour bousculés par l'ouverture des frontières du commerce et la révolution informatique, qui substituent le law shopping au rule of law - poussent partout à leur démantèlement.

La question se pose donc d'un nouveau compromis démocratique permettant aux travailleurs de recouvrer plus de liberté dans la sphère économique sans être privés des garanties et sécurités qu'appelle leur situation de dépendance. L'enquête que nous avons conduite invite à se garder de deux excès contraires dans la recherche d'un tel compris :

- le premier consisterait à transférer sur les entreprises la charge des missions d'intérêt général qui incombe aux États. Certes ce transfert est à bien des égards le prix à payer par les plus grandes entreprises pour le reflux de la puissance publique et la position souveraine qu'elles ont acquise sur la scène mondiale. Mais dans le meilleur des cas, ces entreprises ne pourront être tenues responsables que des impacts sociaux et écologiques de leur activité. Seuls les États demeurent jusqu'à nouvel ordre en charge de la continuité générationnelle de populations données sur un territoire donné ;

- le second excès consisterait au contraire à considérer que, dans une économie sans frontières, la répartition des rôles entre États et entreprises peut demeurer inchangée, et en particulier que ces dernières n'ont toujours pas d'autre responsabilité que d'enrichir leurs actionnaires.

La définition de l'entreprise comme œuvre assignée à l'action des dirigeants et distincte de la réalisation de profits ouvre une voie moyenne raisonnable, qui élargit leur responsabilité sans faire de l'entreprise un erstaz d'État. Un ordre démocratique exige en effet de ses membres la capacité de distinguer son intérêt particulier et l'intérêt commun. Ce qui implique que le pouvoir des dirigeants d'entreprises ne soit pas discrétionnaire, mais assigné à la poursuite de cet intérêt commun, dont il doit répondre à échéances régulières. Et ce qui implique aussi pour les salariés le droit et le devoir de contribuer à la définition et la poursuite de cet intérêt commun. Autrement dit, la démocratisation de l'entreprise suppose que les travailleurs puissent retrouver une certaine maitrise sur l'objet même de leur travail, sur son sens et son contenu.

COURS À L'EXTÉRIEUR

\section{Italie (université de Trento), Faculté de droit}

- «Les bases dogmatiques de la démocratie », le 26 février 2018.

- «La reféodalisation du Droit», le 27 février 2018. 


\section{COLLOQUE - REVISITER LES SOLIDARITÉS EN EUROPE}

À la fin du siècle dernier, Fritz Scharpf avait diagnostiqué l'intégration négative à l'œuvre dans la construction européenne, qui démantèle progressivement les solidarités nationales pour donner libre cours aux libertés économiques, sans être capable d'édifier des solidarités européennes assurant à cette construction légitimité politique et cohésion sociale.

Depuis vingt ans, l'expérience a confirmé le bien-fondé de ce diagnostic. Face à la crise de légitimité partout visible des institutions européennes, la Commission a avancé, aussitôt après le référendum décidant du Brexit, l'idée d'un « socle européen de droit sociaux », qui ne fait aucune place à l'idée de solidarité européenne.

La prophétie néolibérale d'un effacement des solidarités humaines dans l'ordre catallactique d'une Grande société globalisée se trouve pourtant démentie par les faits. Les solidarités ne disparaissent pas, mais elles se déplacent, en sorte que l'affaiblissement de l'État social combiné au manque de solidarité européenne ou internationale suscite le retour à des solidarités de type nationaliste, ethnique ou religieux. La globalisation va aussi de pair avec l'émergence de risques systémiques, qui ont considérablement renforcé des interdépendances bancaires et financières, mais aussi écologiques.

Après avoir dressé un bilan des solidarités mises en œuvre à l'échelle européenne dans quelques domaines clés (tels que la question migratoire, les risques financiers, la fiscalité, les services publics ou l'emploi), le colloque a fait un état des lieux des débats nationaux sur les institutions européennes et permis ainsi de prendre la mesure du poids relatif dans chaque pays du retour à des solidarités nationales et de l'appel à des solidarités européennes renforcées. Il a enfin permis de confronter quelques-uns des projets de sortie de crise avancés dans certains pays.

\section{Introduction}

Ouverture, Alain Supiot: «Les solidarités en Europe : mise en perspective historique », par Jakob Vogel, professeur titulaire de la chaire « Histoire de l'Europe, XIX ${ }^{\mathrm{e}}-\mathrm{XX}^{\mathrm{e}}$ siècles » au Centre d'histoire de Sciences Po.

\section{Les politiques européennes à l'aune de la solidarité}

- «Les injonctions contradictoires en matière migratoire », par Laurence BurgorgueLarsen, professeure de droit public à l'École de droit de la Sorbonne ;

- «Les solidarités européennes en matière fiscale», par Alexandre Maitrot de La Motte, professeur à l'université Paris-Est Créteil ;

- «L'Europe financière et la privatisation de la monnaie», par Gaël Giraud, économiste en chef de l'Agence française de développement, directeur de recherche au CNRS ;

- «Quelle place pour la protection de l'environnement dans la fabrique des normes européennes?», par Béatrice Parance, professeure de droit à l'université UPL Paris 8 Vincennes Saint-Denis ;

- «Entre concurrence et coopération : Europe sociale ou protection par les États? », par Fernando Vasquez, ancien membre de la Direction des affaires sociales de la Commission européenne. 


\section{Vues nationales sur les solidarités en Europe}

- «L'idée de la solidarité européenne dans le pays de Solidarność. D’où viennent les hésitations des Polonais ? », par Marek Safjan, professeur de droit civil à l'université de Varsovie, juge à la Cour de Justice de l'UE, ancien président du Tribunal constitutionnel polonais ;

- «Renaissance du "Politique" en Europe ? Un point de vue allemand », par Ulrich Mückenberger, professeur émérite à l'université de Hambourg et directeur de recherche à l'université de Brême ;

- "Primat de l'économie et activation: la Belgique s'arrime au "modèle allemand" ", par Paul Magnette, professeur de science politique à l'université libre de Bruxelles - ancien ministre président de la Wallonie ;

- «Une vraie solidarité européenne post-crise? ?, par Maria Casas Baamonde, professeure de droit du travail et de la sécurité sociale de l'université Complutense de Madrid, présidente de l'Association espagnole de droit du travail et de la sécurité sociale, ancienne présidente de la Cour constitutionnelle de l'Espagne ;

- «Nouveaux pauvres et réfugiés : les deux pôles actuels de solidarité en Grèce », par Christina Deliyanni Dimitrakou, professeure à la Faculté de droit de l'université Aristote de Thessalonique, secrétaire du Centre de droit économique international et européen ;

- «Perspectives méditerranéennes sur les solidarités en Europe », par Ota De Leonardis, professeure à l'université de Milano Bicocca, directrice de Sui Generis (Centre d'études de sociologie de l'action publique);

- «L'Europe sociale vue de loin : l'expérience portugaise », par Antonio Monteiro Fernandes, professeur à l'Institut universitaire de Lisbonne (ISCTE) ;

- «L'avenir de l'Europe, une perspective suédoise», par Veronika WandDanielsson, Ambassadeur de Suède à Paris ;

- «Brexit et régression du Royaume-Uni hors de la solidarité avec l'Europe ? , par Mark Freedland, professeur émérite de droit du travail à l'université d'Oxford.

\section{Conclusion - Que faire ?}

Table ronde sur les perspectives de refondation (modérateur : Alain Supiot) :

- Michel Aglietta, professeur émérite à l'université Paris-Nanterre, conseiller scientifique au Cepii et à France Stratégie ;

- Franciszek Draus, chercheur indépendant en sciences politiques, spécialiste des questions stratégiques et européennes ;

- Pierre Rodière, professeur émérite à l'université Paris 1, École de droit de la Sorbonne.

\section{RECHERCHE}

\section{PRogRAMMES DE RECHERCHE}

\section{Collaboration scientifique avec la Fondation Charles Léopold Mayer}

Le Collège de France a été lié jusqu'en juin 2018 à la Fondation Charles Léopold Mayer par une convention de recherche, sous la direction d'Alain Supiot et Mireille 
Delmas-Marty, ayant pour objet l'étude des remèdes juridiques à l'irresponsabilité en matière écologique, sociale et financière. Plusieurs manifestations scientifiques ont été organisées dans le cadre de ce programme durant l'année académique 2017 2018 : une journée d'étude sous la direction des professeurs Mireille Delmas-Marty et Alain Wiffels le 27 novembre 2017 ; trois ateliers de recherche le 16 mars, le 9 avril et le 29 juin 2018. Un colloque est prévu pour les 3 et 4 décembre 2018 à la Fondation Hugot du Collège de France.

\section{Recherche «Droit et responsabilité à l'heure de l'anthropocène »}

Placée sous la responsabilité de Luca d'Ambrosio, cette recherche s'inscrit dans un plus large programme qui a pour objectif de fédérer autour des «humanités environnementales » plusieurs chercheurs des grands établissements parisiens réunis au sein de l'université Paris Sciences et Lettres. Deux manifestations scientifiques ont été organisées dans le cadre de ce programme de recherche: deux journées d'étude sur la responsabilité des entreprises les 19 et 20 octobre 2017 ; un colloque pluridisciplinaire « The right use of the earth: Knowledge, power and duties » du 29 mai au $1^{\text {er }}$ juin 2018 (www.rightuseofearth.sciencesconf.org).

\section{ACTIVITÉS DES CHERCHEURS RATTACHÉS À LA CHAIRE}

\section{Chercheurs contractuels}

Linxin He est docteur en droit, attaché de recherche.

Publications :

HE L., Droits sociaux fondamentaux et droit de l'Union européenne, thèse de doctorat sous la direction d'Étienne Pataut à l'université Panthéon-Sorbonne, 2017, synthèse publiée in : Revue trimestrielle de droit européen, 2018, p. 25-44.

Luca d'Ambrosio est docteur en droit et chargé de recherche contractuel (convention avec la Fondation Charles Léopold Mayer).

Publications :

D’ambrosio L., Giudicelli-Delage G. et Manacorda S. (dir.), Principe de précaution et métamorphoses de la responsabilité, Paris, Mare \& Martin, coll. « Collection de l'Institut des sciences juridique et philosophique de la Sorbonne », $\mathrm{n}^{\circ}$ 44, 2018.

D'Ambrosio L. et VozzA D., «Lieto fine pour l'affaire Taricco ou le résultat du dialogue constructif entre la Cour constitutionnelle italienne et la Cour de justice de l'Union européenne », Revue trimestrielle de droit européen, Chronique «Jurisprudences nationales intéressant le droit de l'Union européenne », n 1, 2018, p. 173-178.

Camila Perruso est doctorante et attachée de recherche contractuelle (convention avec la Fondation Charles Léopold Mayer).

Publications :

Perruso C. et Martin-Chenut K., «Les perspectives d'appréhension du principe de précaution par le système interaméricain des droits de l'homme », in L. D'AMBrosio, G GiUdiCELli-DelaGE et S. MANACORDA (dir.), Principe de précaution et métamorphoses de la responsabilité, Paris, Mare \& Martin, "Collection de l'Institut des sciences juridique et philosophique de la Sorbonne », $\mathrm{n}^{\circ}$ 44, 2018, p. 105-118. 
PERRUSO C., «Notas acerca da evolução jurisprudencial do direito ao meio ambiente nos mecanismos regionais de proteção dos direitos humanos », in S. GARCÍA MUÑOZ et C.B. LEAL (dir.), Gênero, meio ambiente e direitos humanos, Fortaleza, Expressão gráfica e editora, 2017, p. 29-50.

PERRUSO C., «Perspectivas da informação ambiental no sistema interamericano de direitos humanos », in P. GAUTREAU et N. MonebhurRun (dir.), Direito à Informação Ambiental : uma agenda de pesquisa interdisciplinar, Paraná, Prismas, 2017, p. 293-312, https://halshs. archives-ouvertes.fr/halshs-01728227/document.

\section{Chercheurs invités}

Andrea Allamprese est professeur à l'université de Modène et Reggio Emilia.

AllAmprese A., «Le invenzioni del lavoratore autonomo », in M. LANOTTE (dir.), Il lavoro autonomo e occasionale, Milan, Giuffrè, 2018, p. 129-148.

AllAmprese A., «La tutela della salute e della sicurezza del lavoratore "agile" », Rivista giuridica del lavoro e della previdenza sociale, $\mathrm{n}^{\mathrm{0}}$ 2, 2017, p. 307-330.

Allamprese A., «Il licenziamento dei dipendenti delle pubbliche amministrazioni davanti alla Corte di Cassazione », Rivista giuridica del lavoro, 2017, $\mathrm{n}^{\circ}$ 1, p. 78-90.

Konstantina Papathanasiou est maître de conférences à la Faculté de droit de Ratisbonne.

PAPATHANASIOU K., « Environmental criminal law : A vital part of the regulatory framework », in K. MATHIS et B.R. HUbER (dir.), Environmental Law and Economics, Cham, Springer International Publishing, coll. « Economic Analysis of Law in European Legal Scholarship », $n^{0} 4$, 2017, p. 513-526, DOI : 10.1007/978-3-319-50932-7_21.

PAPATHANASIOU K., "Vorsatz und Fahrlässigkeit beim unerlaubten Handeltreiben mit Betäubungsmitteln : Zugleich Besprechung von BGH 1 StR 64/17, 20 septembre 2017 », HöchstRichterliche Rechtsprechung im Strafrecht (HRRS), n 66, 2018.

PAPATHANASIOU K., «Eigenverantwortung, Neuronensteuerung oder Habitus? Der homo autonomus et inspiratus als strafrechtliches Menschenbild», in A. FUNKE (dir.), Menschenbilder im Recht, Erlangen-Nürnberg, Mohr Siebeck Verlag, 2017.

\section{PuBlicATIONS}

\section{LIVRES}

SuPIOT A. (dir.), Face à l'irresponsabilité : la dynamique de la solidarité, Paris, Éditions du Collège de France, coll. «Conférences », 2018, et en ligne sur OpenEditions Books. DOI : 10.4000/books.cdf.5188.

\section{ARTICLES}

SuPIOT A., «Réflexions sur le dépérissement de l'État », LEX Social, Revista juridica de les Derechos Sociales, vol. 8, n 1, 2018, p. 365-381.

\section{TRADUCTIONS}

SuPIOT A., Governance by Numbers. The Making of a Legal Model of Allegiance, trad. S. BROWN, Oxford/Portland, Hart Publishing, coll. « Hart studies in comparative public law », 2017 (traduction anglaise de La Gouvernance par les nombres. Cours au Collège de France, 2012-2014, Paris, Fayard, 2015). 
SUPIOT A., 法的人間 ホモ・ジュリディクス: 法の人類学的機能, trad. K. HASHIMOTO et S. DAKE, Tokyo, Keiso Shobo, 2018 (traduction japonaise de Homo juridicus. Essai sur la fonction anthropologique du Droit, Paris, Seuil, 2005).

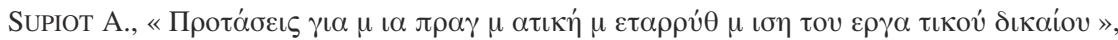

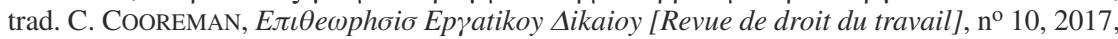
p. 1279-1310 (traduction grecque de «Les voies d'une vraie réforme du droit du travail», préface à la $2^{\mathrm{e}}$ édition de Au-delà de l'emploi, Paris, Flammarion, 2016).

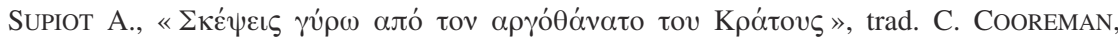

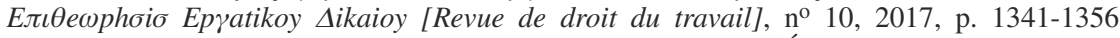
(traduction grecque de "Réflexions sur le dépérissement de l'État», LEX Social, Revista juridica de les Derechos Sociales, vol. 8, $\mathrm{n}^{\circ}$ 1, 2018, p. 365-381).

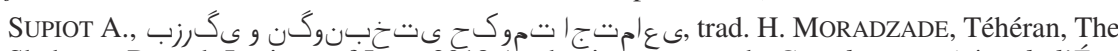
Shahr -e- Danesh Institute of Law, 2018 (traduction persane de Grandeur et misère de l'État social, Paris, Collège de France/Fayard, 2013).

\section{ENTRETIENS ET ARTICLES DE PRESSE}

SuPIOT A., «De la citoyenneté économique », entretien avec Thibault Le Texier, Esprit, no 3 , mars 2018, p. 52-63, DOI : 10.3917/espri.1803.0052.

SuPIOT A. et LA PORTE X. de, « Entretien sur Simone Weil », L'Obs, n 2751, 27 juillet 2017, L'été des philosophes $2 / 5$, p. 56-59. Traduction anglaise : « The Perspective of the Drowning: Alain Supiot on Simone Weil », trad. David Broder, Verso, 2017.

Supiot A., «A Labour code for the 21st century », Le Monde diplomatique, English edition, mai 2018 (traduction anglaise de «Et si l'on refondait le droit du travail...», Le Monde diplomatique, octobre 2017, p. 22-23. 